\title{
Genistein inhibits A549 human lung cancer cell proliferation via miR-27a and MET signaling
}

\author{
YANG YANG $^{1 *}$, AIMIN ZANG $^{1 *}$, YOUCHAO JIA ${ }^{1}$, YANHONG SHANG $^{1}$, ZHUOQI ZHANG $^{1}$, \\ $\mathrm{KUN} \mathrm{GE}^{2}$, JINCHAO ZHANG ${ }^{2}$, WUFANG FAN ${ }^{3}$ and BEI WANG ${ }^{3}$
}

${ }^{1}$ Department of Medical Oncology, Hebei Key Laboratory of Cancer Radiotherapy and Chemotherapy, Affiliated Hospital of Hebei University, Baoding, Hebei 071000; ${ }^{2}$ College of Chemistry and Environmental Science, Key Laboratory of Chemical Biology of Hebei Province; ${ }^{3}$ College of Life Sciences, Hebei University, Baoding, Hebei 071002, P.R. China

Received February 11, 2015; Accepted March 3, 2016

DOI: $10.3892 / \mathrm{ol} .2016 .4817$

\begin{abstract}
Genistein is a soybean isoflavone; in its aglycone it has various biological activities. Animal experiments, clinical studies and epidemiological investigations suggest that genistein has preventative and curative functions for a number of diseases, particularly in cancer. The present study explored the potential anti-cancer effect of genistein by observing its role in inhibiting A549 human lung cancer cell proliferation and investigating the possible mechanism. A549 cells were exposed to various concentrations of genistein $(0,10,25,50$, 100 and $200 \mu \mathrm{M}$; dissolved in physiological saline) for 1, 2 and 3 days. Subsequently, the viability of A549 cells was determined by 3-(4,5-dimethylthiazol-2-yl)-2,5-diphenyltetrazolium bromide assay, cell apoptosis was examined using a flow cytometer, caspase 3/9 activity was measured using commercial kits, reverse transcription quantitative polymerase chain reaction was used to analyze the miR-27a expression and western blotting was used to investigate MET protein expression. The results suggested a significant inhibition of A549 cell growth following treatment with genistein in a time- and dose-dependent manner. The current study also indicated that treatment with genistein significantly induces cell apoptosis and promotes caspase-3/9 activation of A549 cells in a dose-dependent manner. Further functional assays revealed that the anti-cancer effect of genistein activated microRNA-27a (miR-27a) expression levels and reduced MET protein expression in A549 cells. In conclusion, the present
\end{abstract}

Correspondence to: Mr. Youchao Jia, Department of Medical Oncology, Hebei Key Laboratory of Cancer Radiotherapy and Chemotherapy, Affiliated Hospital of Hebei University, 212 Yuhua East Road, Baoding, Hebei 071000, P.R. China

E-mail: youchaojia@yeah.net

${ }^{*}$ Contributed equally

Key words: genistein, lung cancer, microRNA-27a, MET proto-oncogene study demonstrates that genistein inhibits A549 human lung cancer cell proliferation. Furthermore, this study reports, for the first time, a correlation between the anti-cancer effect of genistein and miR-27a-mediated MET signaling.

\section{Introduction}

Non-small lung cancer (NSCLC) has high morbidity and mortality rates; according to the latest data, 222,520 new cases and 157,300 mortalities were reported in USA in 2010. NSCLC is the leading cause of cancer-related mortality in men and women. According to 2010 Ministry of Health data, lung cancer rose from the third most common to the most common cause of cancer-related mortality in 1992, and reached a rate of 30.83/100,000 individuals. Trends of rising incidence and younger age have become more obvious, raising greater concern $(1,2)$. There is a close association between smoking and lung carcinoma; in addition, atmospheric pollution and automobile exhaust fumes are also lung cancer risk factors. Furthermore, chronic bronchitis, pulmonary heart disease, pulmonary fibrosis, pulmonary tuberculosis and pneumoconiosis are associated with the incidence of lung cancer $(3,4)$.

MicroRNA (miRNA/miR) is a small single-stranded RNA ( 22 nucleotides) that occurs in eukaryotes. miRNA does not have the function of the encoded protein; instead, its biological function is to pair with the 3'-untranslated region of complementary target mRNAs. If the miRNA is fully complementary it will cause target mRNA degradation; if the miRNA complementarity is incomplete, it may prevent the target mRNA translating into protein (5). Bioinformatic research has shown that miRNA constitutes $1-5 \%$ of the total number of animal genes, and $\sim 60 \%$ encoding genes in animals are regulated by miRNA. Thus, miRNA is a gene regulation factor that widely exists and has an important role in living organisms (6). Experimental research has also demonstrated that miRNAs are key in various biological activities, such as cell proliferation and differentiation, apoptosis, generation, migration, invasion and metastasis of tumor cells, resistance mechanisms and the antivirus effect. Numerous miRNAs can promote or inhibit metastasis (7). Thus, miRNAs may become novel targets for cancer treatment. 
MET proto-oncogene, which encodes the MET protein, is a type of tyrosine kinase activity growth factor receptor that can specifically bind to hepatocyte growth factor (HGF) and activate its downstream signal transduction pathways, regulating cell proliferation, energy, migration, angiogenesis, invasion and morphogenesis (8). The tyrosine kinase encoded by MET can adjust the invasive growth of tumor cells, promoting the occurrence and development of lung tumors (9). Normal MET signaling pathways regulate embryonic development and tissue damage repair, whereas abnormal activation promotes tumor metastasis. MET exhibits abnormal expression in a variety of lung carcinoma tumor tissues and it is closely associated with tumor progression (10).

Genistein is an antimicrobial toxin precursor that predominantly exists in the biosynthesis of leguminous plants; it is the active component and has various physiological functions (11). It is an effective antioxidant and protein tyrosine activation enzyme inhibitor, as well as a plant estrogen. Genistein also has extensive pharmacological effects in animal cells. A previous study found that genistein has anticancer effects, including in vitro antitumor effects in human breast cancer cell lines (12) and prostate cancer cells (13). Thus, genistein is a potential cancer chemoprevention agent with a broad development prospect (14). In the present study, we hypothesized that the potential anti-cancer effect of genistein would inhibit A549 human lung cancer cell proliferation via miR-27a and MET signaling.

\section{Materials and methods}

Chemicals. RPMI-1640 medium and fetal bovine serum were obtained from Thermo Fisher Scientific, Inc. (Waltham, MA, USA). 3-(4,5-dimethylthiazol-2-yl)-2,5-diphenyltetrazolium bromide (MTT) was obtained from Sigma-Aldrich (St. Louis, MO, USA). Annexin V-fluorescein isothiocyanate (FITC)/propidium iodide (PI) apoptosis assay kit was obtained from Nanjing KeyGen Biotech Co., Ltd. (Nanjing, China).

Cell lines and cell growth. The A549 human lung cancer cell line was provided by Hebei University (Baoding, China), and maintained in RPMI-1640 medium with $10 \%$ fetal bovine serum, $100 \mathrm{U} / \mathrm{ml}$ penicillin and $100 \mathrm{mg} / \mathrm{ml}$ streptomycin (Sangon Biotech Co., Ltd., Shanghai, China) in a $37^{\circ} \mathrm{C}$ incubator with $5 \% \mathrm{CO}_{2}$.

Cell viability. The in vitro effect of genistein on the viability of A549 cells was determined by MTT assay. Briefly, A549 cells were plated onto a 96-well plate at a density of $1 \times 10^{4}$ cells/well. Subsequently, A549 cells were exposed to different concentrations of genistein $(0,10,25,50,100$ and $200 \mu \mathrm{M}$; Sigma-Aldrich; dissolved in physiological saline) for 1, 2 and 3 days. Subsequently, $20 \mu \mathrm{l}$ of $0.5 \%$ MTT solution with phosphate-buffered saline (Beyotime Institute of Biotechnology, Haimen, China) was added to each well and incubated for $4 \mathrm{~h}$ at $37^{\circ} \mathrm{C}$ with $5 \% \mathrm{CO}_{2}$. After the incubation period, the culture medium was replaced, $200 \mu 1$ of dimethyl sulfoxide (Sinopharm Chemical Reagent Co., Ltd., Shanghai, China) was added to each well and shaken for $20 \mathrm{~min}$ at room temperature. The optical density of each well was determined with a spectrophotometer at $492 \mathrm{~nm}$ (Varioskan Flash microplate reader; Thermo Fisher Scientific, Inc.).

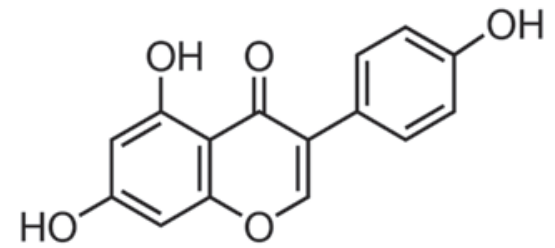

Figure 1. Chemical structure of genistein.

Annexin V-FITC/PI apoptosis assay. The in vitro effect of genistein on A549 cell viability was determined by performing an Annexin V-FITC/PI assay. Briefly, A549 cells were plated onto a 6 -well plate at a density of $1 \times 10^{6}$ cells/well. Subsequently, A549 cells were exposed to different concentrations of genistein $(0,25,50$ and $100 \mu \mathrm{M})$ for 2 days. A total of $5 \mu \mathrm{l}$ Annexin V-FITC $(5 \mu \mathrm{g} / \mathrm{ml})$ and $5 \mu \mathrm{l}$ PI $(1 \mu \mathrm{g} / \mathrm{ml})$ was added and incubated for $10 \mathrm{~min}$ at room temperature in the dark. Then, cell apoptosis was examined using a flow cytometer (FC 500; Beckman Coulter, Inc., Brea, CA, USA).

Analysis of caspase-3/9 activity. Following plating and genistein treatment, as described for the Annexin V-FITC/PI assay, A549 cells were incubated with cell lysis buffer (Beyotime Institute of Biotechnology) for $30 \mathrm{~min}$ on ice and then centrifuged at $12,000 \mathrm{x} \mathrm{g}$ for $10 \mathrm{~min}$ at $4^{\circ} \mathrm{C}$. The protein concentrations of the cell solutions were determined using a Pierce BCA protein assay kit (Thermo Fisher Scientific, Inc.). Equal quantities of protein were mixed with $2 \mathrm{mM}$ Ac-LEHD-pNA for caspase-9 and $2 \mathrm{mM}$ Ac-DEVD-pNA for caspase-3 (Beyotime Institute of Biotechnology). The mixtures were incubated at $37^{\circ} \mathrm{C}$ for $2 \mathrm{~h}$ in the dark and detected at a wavelength of $405 \mathrm{~nm}$ (Varioskan Flash microplate reader).

Reverse transcription-quantitative polymerase chain reaction (RT-qPCR). Following plating and genistein treatment, as described for the Annexin V-FITC/PI assay, total RNA was extracted from the A549 cells using TRIzol reagent (Invitrogen; Thermo Fisher Scientific, Inc.). RNA (1-2 $\mu \mathrm{g})$ was reverse transcribed to synthesize complementary DNA using the SuperScript III First-Strand Synthesis System (Thermo Fisher Scientific, Inc.) miR-27a was specifically amplified and its expression was quantified by performing TaqMan miRNA RT-qPCR assays (Applied Biosystems; Thermo Fisher Scientific, Inc.), according to the manufacturer's protocol. The PCR cycling conditions were as follows: $5 \mathrm{~min}$ at $94^{\circ} \mathrm{C}, 30 \mathrm{sec}$ at $94^{\circ} \mathrm{C}, 30 \mathrm{sec}$ at $60^{\circ} \mathrm{C}$ and $30 \mathrm{sec}$ at $72^{\circ} \mathrm{C}$ (for a total of 40 cycles), followed by a cycle of $5 \mathrm{~min}$ at $72^{\circ} \mathrm{C}$. miR-27a levels were assessed by SYBR Green qPCR (Takara Biotechnology Co., Ltd., Dalian, China). The forward $(\mathrm{F})$ and reverse $(\mathrm{R})$ primers were used were as follows: F, 5'-TCCGTGAGAGCTGGAAAACC-3' and R, 5'-TGGTTC TAACTA ACTCCAGCCG-3' for miR-27a; F, 5'-CGCTTC GGCACATATACTA-3' and R, 5'-CGCTTCACGAATTTG CGTGTCA-3' for U6. Analysis of mRNA expression of miR $27 \mathrm{a}$ was calculated according to the $2-\Delta \Delta \mathrm{Cq}$ method (15).

Western blotting. After plating and genistein treatment, as described for the Annexin V-FITC/PI assay, A549 cells were incubated with $500 \mu \mathrm{l}$ RIPA lysis buffer for $30 \mathrm{~min}$ on ice and centrifuged at $12,000 \mathrm{x}$ g for $10 \mathrm{~min}$ at $4^{\circ} \mathrm{C}$. The protein 

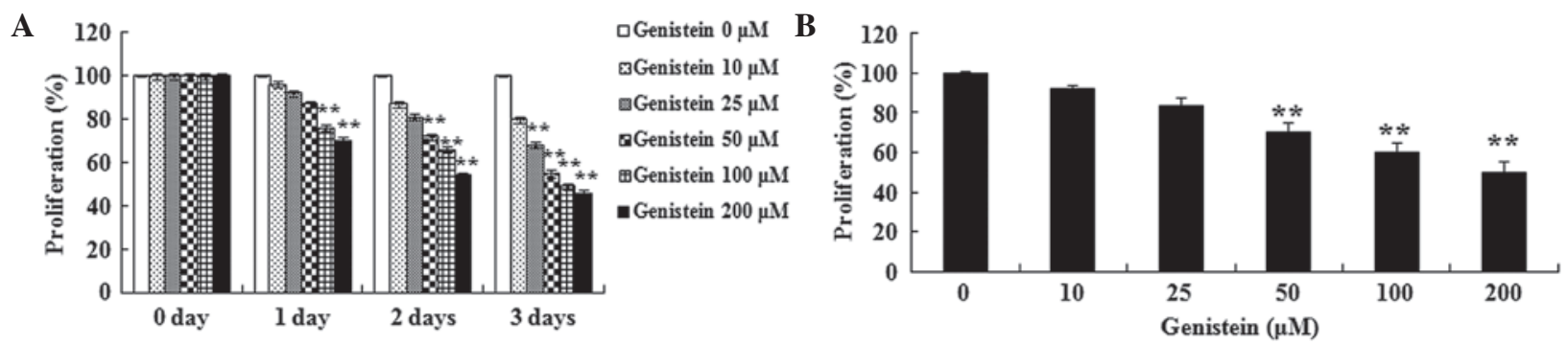

Figure 2. In vitro effect of genistein on A549 cell viability following treatment for (A) 1-3 and (B) 2 days. Results are presented as mean \pm standard deviation. ${ }^{* *} \mathrm{P}<0.01$ vs. $0 \mu \mathrm{M}$ genistein.

concentrations of the cell solutions were determined using a Pierce BCA protein assay kit (Thermo Fisher Scientific, Inc.). Equal quantities $(80 \mu \mathrm{g})$ of protein was resolved on $12 \%$ SDS-PAGE gel and transferred to nitrocellulose membrane (Santa Cruz Biotechnology, Inc., CA, USA). Subsequently, the membranes were blocked with non-fat milk in Tris-buffered saline with Tween 20 and incubated with polyclonal rabbit MET (1:1,000; catalog no., sc-8307; Santa Cruz Biotechnology, Inc.) and $\beta$-actin (1:500; catalog no., D110007; Sangon Biotech Co., Ltd.) primary antibodies overnight at $4^{\circ} \mathrm{C}$. The membranes were incubated with horseradish peroxidase-conjugated mouse anti-rabbit IgG secondary antibodies (1:5,000; catalog no., M1003-7; Hangzhou Hua'an Biological Technology Co., Ltd., Hangzhou, China) for $1 \mathrm{~h}$ at $37^{\circ} \mathrm{C}$. Membranes were visualized using an Enhanced Chemiluminescence detection kit (Beyotime Institute of Biotechnoloy). The bands were quantified by densitometric analysis using Image J software (version 1.44p; National Institutes of Health, Bethseda, MD, USA).

Statistical analysis. Statistical analysis was performed using SPSS statistical software (version 19.0; IBM SPSS, Chicago, IL, USA) and results are presented as the mean \pm standard deviation of three replicate experiments. Statistical differences between the control and treatment samples were determined by Student's t-test. Differences among experimental groups were evaluated by one-way analysis of variance. $\mathrm{P}<0.05$ was considered to indicate a statistically significant.

\section{Results}

In vitro effect of genistein on A549 cell viability. The chemical structure of genistein (Sigma-Aldrich; with a purity of 98\%) is indicated in Fig. 1. In the present study, genistein was dissolved in physiological saline. The present study investigated the possible anti-cancer effect of genistein on the viability of A549 cells by performing an MTT assay. Fig. 2A and B demonstrates that the treatment with genistein inhibited the viability of A549 cells in a time- and dose-dependent manner. The results suggest that A549 cell viability was significantly inhibited by different concentrations of genistein $(25,50,100$ and $200 \mu \mathrm{M})$ for 3 days $(\mathrm{P}<0.01)$. When the treatment was reduced to 2 days, the viability of A549 cells was significantly inhibited by 50, 100 and $200 \mu \mathrm{M}$ genistein ( $\mathrm{P}<0.01$; Fig. 2B). Furthermore, A549 cell viability was significantly suppressed following treatment with 100 and $200 \mu \mathrm{M}$ genistein for a period of 1 day $(\mathrm{P}<0.01)$. These data suggest that genistein may significantly inhibit human lung cancer cell viability.

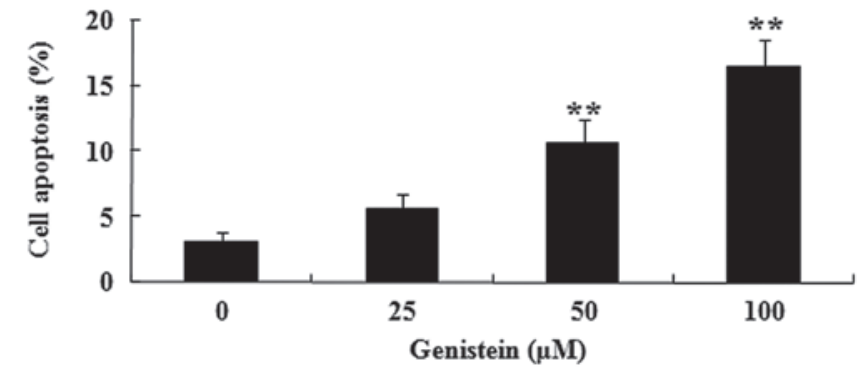

Figure 3. In vitro effect of genistein on cell apoptosis in A549 cells. Results are presented as mean \pm standard deviation. ${ }^{* *} \mathrm{P}<0.01$ vs. $0 \mu \mathrm{M}$ genistein.

In vitro effect of genistein on A549 cell apoptosis. To determine the possible anti-cancer effect of genistein on A549 cell apoptosis, the present study measured the apoptosis of A549 cells by performing an Annexin V-FITC/PI apoptosis assay. Fig 3. indicates that administration of genistein (50 and $100 \mu \mathrm{M})$ significantly induces the apoptosis of A549 cells in dose-dependent manner $(\mathrm{P}<0.01)$. These data suggest that genistein may induce human lung cancer cell apoptosis.

In vitro effect of genistein on caspase-3/9 activation in A549 cells. The present study aimed to investigate the possible anti-cancer effect of genistein on caspase-3/9 activation in A549 cells using commercially available kits. As indicated in Fig. 4A and B, pretreatment with genistein $(50$ and $100 \mu \mathrm{M})$ for 2 days significantly promoted caspase- 3 and -9 activation in A549 cells, respectively $(\mathrm{P}<0.01)$. These data suggest that genistein may promote human lung cancer cell apoptosis by activation of the caspase-3/9 signaling pathways.

In vitro effect of genistein on miR-27a expression in A549 cells. To explain the possible anti-cancer effect of genistein on miR-27a expression in human lung cancer, miR-27a expression of A549 cell was analyzed by RT-qPCR. As presented in Fig. 5, after 2 days of treatment with genistein (50 and $100 \mu \mathrm{M})$, miR-27a expression in A549 cells was significantly increased $(\mathrm{P}<0.01)$. These data indicate that genistein may activate miR-27a expression levels in human lung cancer.

In vitro effect of genistein on MET protein expression in A549 cells. To investigate the possible anti-cancer effect of genistein on MET protein expression in human lung cancer, the present study determined MET protein expression levels in A549 cells by performing western blotting. As shown in Fig. 6A and B, the effect of incubation with genistein (50 and 

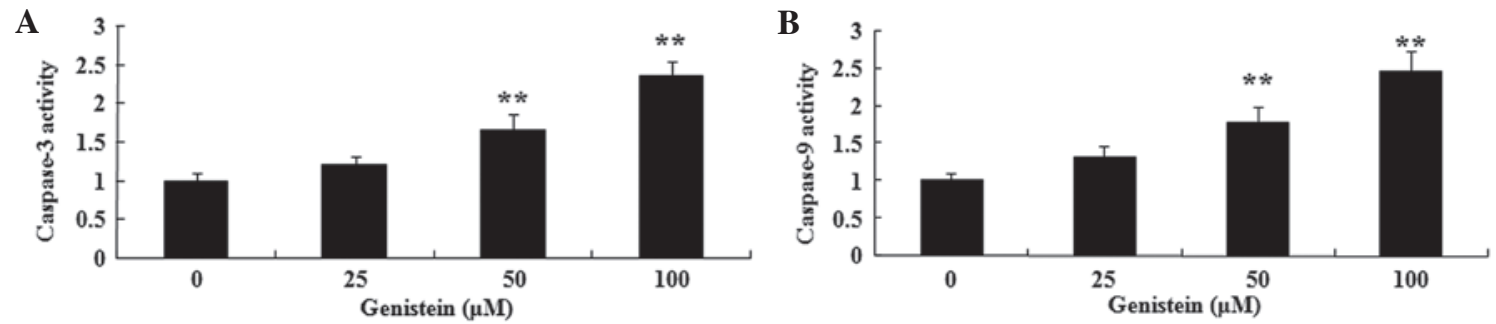

Figure 4. In vitro effect of genistein on (A) caspase-3 and (B) casepase-9 activation in A549 cells. Results are presented as mean \pm standard deviation. ${ }^{* *} \mathrm{P}<0.01$ vs. $0 \mu \mathrm{M}$ genistein.

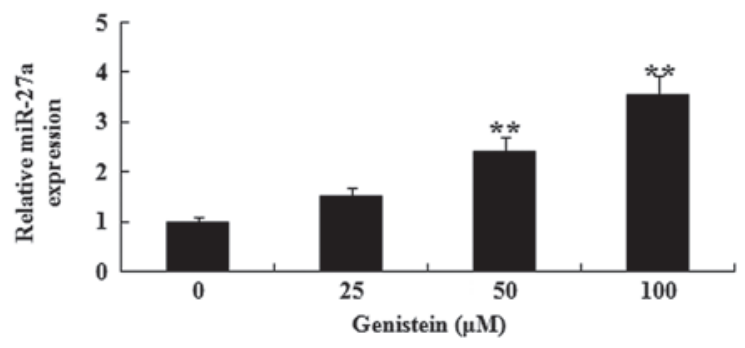

Figure 5. In vitro effect of genistein on miR-27a expression in A549 cells. Results are presented as mean \pm standard deviation. ${ }^{* *} \mathrm{P}<0.01 \mathrm{vs} .0 \mu \mathrm{M}$ genistein.

A

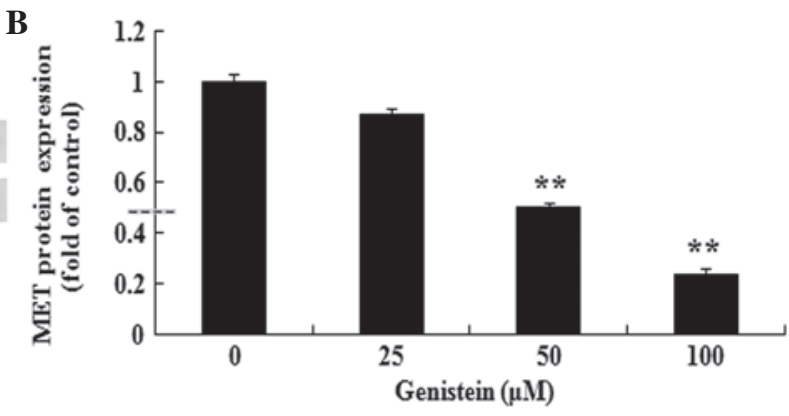

Figure 6. In vitro effect of genistein on MET protein expression in A549 cells. The effect of genistein on MET protein expression using (A) western blot analysis and (B) statistical analysis of MET protein expression level of A549 cell. Results are presented as mean \pm standard deviation. ${ }^{* *} \mathrm{P}<0.01 \mathrm{vs} .0 \mu \mathrm{M}$ genistein.

$100 \mu \mathrm{M})$ for 2 days significantly reduced MET protein expression in A549 cells $(\mathrm{P}<0.01)$. These data suggest that genistein may decrease MET protein expression levels in human lung cancer.

\section{Discussion}

NSCLC has the highest morbidity and mortality rates of all malignant tumors in the majority of the world's countries (16). Approximately two-thirds of patients are diagnosed with locally advanced disease or distant metastasis at the time of diagnosis, thus, the opportunity for surgery is not possible. Instead, the primary treatment of advanced NSCLC is systemic chemotherapy treatment, however, the associated analysis indicates that the curative effect of chemotherapy on advanced NSCLC has entered a plateau period. The use of radiation therapy and biological treatment are also poor at improving its curative effect (17). Therefore, the identification of effective treatments is currently the top priority in lung cancer research. To the best of our knowledge, the present study is the first to demonstrate that genistein inhibits viability, induces apoptosis and promotes caspase-3/9 activation of A549 cells in a dose-dependent manner. Similarly, Xiao et al (18) demonstrated that genistein inhibits human colorectal cancer metastasis. Suzuki et al (19) also reported that genistein induces the apoptosis of human pancreatic cancer cells. These data suggest that genistein may be a potential targeting drug for human lung cancer.

The occurrence and development of lung cancer is a complex, multiple links process, including the oncogene activation and mutations or deficiency of tumor suppressor genes, in the process of the occurrence and metastasis of lung carcinoma, genetic variation is the extensive research focus for many years (20). miRNA widely exists in human chromosomes, and it have very strong organization specificity. Based regulation on proliferation, differentiation, and apoptosis process of cells, miRNA can regulate tumor biological behavior, affect the occurrence and development of lung carcinoma. Therefore, targeted therapy to the miRNA has the vital significance in the gene therapy of lung carcinoma. Acunzo et al (21) reported that miR-27a can regulate MET and EGFR in NSCLC. In the current study, genistein activated miR-27a expression of NSCLC. Xia et al (22) demonstrated that genistein inhibits cell growth of pancreatic cancer cells through regulation of miR-27a and human uveal melanoma cells (23). 
MET protein is the receptor of hepatocyte growth factor/scatter factor (HGF/SF). MET as the proto-oncogene, the product has tyrosine kinase activity after it is activated, HGF/SF specificity combine with it, activate a series of trans-membrane signal transduction pathways, thus stimulate epithelial cell morphogenesis, proliferation and migration (24). Research has demonstrated that the MET gene has excessive expression in various types of malignant tumor, such as lung and breast carcinoma. Under normal circumstances, MET signaling pathways involve in embryogenesis, while abnormal MET signaling pathways are closely associated with tumor occurrence and development; in particular, MET has an important role in promoting tumor cell invasion and metastasis (25). Increased MET expression appears to be a prognostic factor in patients with NSCLC following curative surgery (26). In the present study, genistein was observed to decrease MET protein expression levels in human lung cancer. Similarly, Singletary and Ellington (27) reported that genistein suppresses the proliferation of human breast epithelial cells through downregulation of the proto-oncogene MET.

In conclusion, the present study demonstrates for the first time that genistein inhibits A549 human lung cancer cell proliferation via miR-27a and MET signaling. Additional studies are required to clarify the mechanism underlying the interaction between the miRNAs and the potential anti-cancer effect of genistein; in particular, it is important to determine the molecular mechanisms of genistein in cancer.

\section{Acknowledgements}

The present study was supported by Hebei University Special Funds for Medical Science Construction Project (grant no., 2015B1001).

\section{References}

1. Chen Q, Cheng P, Yin T, He H, Yang L, Wei Y and Chen X: Therapeutic potential of bone marrow-derived mesenchymal stem cells producing pigment epithelium-derived factor in lung carcinoma. Int J Mol Med 30: 527-534, 2012.

2. Chen X, Ni J, Meng H, Li D, Wei Y, Luo Y and Wu Y: Interleukin15: A potent adjuvant enhancing the efficacy of an autologous whole-cell tumor vaccine against Lewis lung carcinoma. Mol Med Rep 10: 1828-1834, 2014.

3. Tatematsu T, Sasaki H, Shimizu S, Okuda K, Shitara M, Hikosaka Y, Moriyama S, Yano M, Brown J and Fujii Y: Investigation of neurotrophic tyrosine kinase receptor 1 fusions and neurotrophic tyrosine kinase receptor family expression in non-small-cell lung cancer and sensitivity to AZD7451 in vitro. Mol Clin Oncol 2: 725-730, 2014.

4. Lo HM, Shieh JM, Chen CL, Tsou CJ and Wu WB: Vascular endothelial growth factor induces CXCL1 chemokine release via JNK and PI-3K-dependent pathways in human lung carcinoma epithelial cells. Int J Mol Sci 14: 10090-10106, 2013.

5. Macchiaroli N, Cucher M, Zarowiecki M, Maldonado L, Kamenetzky L and Rosenzvit M: microRNA profiling in the zoonotic parasite Echinococcus canadensis using a high-throughput approach. Parasit Vectors 8: 83, 2015.

6. Yang X, Zhong J, Ji Y, Li J, Jian Y, Zhang J and Yang W: The expression and clinical significance of microRNAs in colorectal cancer detecting. Tumour Biol 36: 2675-2684, 2015.

7. Lerebours F, Cizeron-Clairac G, Susini A, Vacher S, Mouret-Fourme E, Belichard C, Brain E, Alberini JL, Spyratos F, Lidereau R and Bieche I: miRNA expression profiling of inflammatory breast cancer identifies a 5-miRNA signature predictive of breast tumor aggressiveness. Int J Cancer 133: 1614-1623, 2013
8. Gherardi E, Birchmeier W, Birchmeier C and Vande Woude G: Targeting MET in cancer: Rationale and progress. Nat Rev Cancer 12: 89-103, 2012.

9. Huang L, An SJ, Chen ZH, Su J, Yan HH and Wu YL: MET expression plays differing roles in non-small-cell lung cancer patients with or without EGFR mutation. J Thorac Oncol 9: 725-728, 2014.

10. Tsuda M, Davis IJ, Argani P, Shukla N, McGill GG, Nagai M, Saito T, Laé M, Fisher DE and Ladanyi M: TFE3 fusions activate MET signaling by transcriptional up-regulation, defining another class of tumors as candidates for therapeutic MET inhibition. Cancer Res 67: 919-929, 2007.

11. Han S, Wu H, Li W and Gao P: Protective effects of genistein in homocysteine-induced endothelial cell inflammatory injury. Mol Cell Biochem 403: 43-49, 2015.

12. Pons DG, Nadal-Serrano M, Blanquer-Rossello MM, Sastre-Serra J, Oliver J and Roca P: Genistein modulates proliferation and mitochondrial functionality in breast cancer cells depending on ERalpha/ERbeta ratio. J Cell Biochem 115: 949-958, 2014.

13. Pavese JM, Krishna SN and Bergan RC: Genistein inhibits human prostate cancer cell detachment, invasion and metastasis. Am J Clin Nutr 100 (Suppl 1): S431-S436, 2014.

14. Spoerlein C, Mahal K, Schmidt H and Schobert R: Effects of chrysin, apigenin, genistein and their homoleptic copper (II) complexes on the growth and metastatic potential of cancer cells. J Inorg Biochem 127: 107-115, 2013.

15. Livak and Schmittgen: Analysis of relative gene expression data using real-time quantitative PCR and the $2-\Delta \Delta \mathrm{Ct}$ method. Methods 25: 402-408, 2001.

16. Ishii H, Azuma K, Yamada K, Kinoshita T, Imamura Y and Hoshino T: Predictive factors in patients with EGFR mutation-negative non-small cell lung cancer treated with erlotinib. Oncol Lett 8: 2699-2704, 2014.

17. Jiang L, Liang X, Liu M, Wang W, Ma J, Guo Q, Han L, Yang C and Nan K: Reduced expression of liver kinase B1 and Beclin1 is associated with the poor survival of patients with non-small cell lung cancer. Oncol Rep 32: 1931-1938, 2014.

18. Xiao X, Liu Z, Wang R, Wang J, Zhang S, Cai X, Wu K, Bergan RC, Xu L and Fan D: Genistein suppresses FLT4 and inhibits human colorectal cancer metastasis. Oncotarget 6: 3225-3239, 2015

19. Suzuki R, Kang Y, Li X, Roife D, Zhang R and Fleming JB: Genistein potentiates the antitumor effect of 5-Fluorouracil by inducing apoptosis and autophagy in human pancreatic cancer cells. Anticancer Res 34: 4685-4692, 2014.

20. Capodanno A, Boldrini L, Proietti A, Alì G, Pelliccioni S, Niccoli C, D'Incecco A, Cappuzzo F, Chella A, Lucchi M, et al: Let-7 $\mathrm{g}$ and miR-21 expression in non-small cell lung cancer: Correlation with clinicopathological and molecular features. Int J Oncol 43: 765-774, 2013.

21. Acunzo M, Romano G, Palmieri D, Laganá A, Garofalo M, Balatti V, Drusco A, Chiariello M, Nana-Sinkam P and Croce CM: Cross-talk between MET and EGFR in non-small cell lung cancer involves miR-27a and Sprouty2. Proc Natl Acad Sci USA 110: 8573-8578, 2013.

22. Xia J, Cheng L, Mei C, Ma J, Shi Y, Zeng F, Wang Z and Wang Z: Genistein inhibits cell growth and invasion through regulation of miR-27a in pancreatic cancer cells. Curr Pharm Des 20: 5348-5353, 2014

23. Sun Q, Cong R, Yan H, Gu H, Zeng Y, Liu N, Chen J and Wang B: Genistein inhibits growth of human uveal melanoma cells and affects microRNA-27a and target gene expression. Oncol Rep 22: 563-567, 2009

24. Freudlsperger C, Alexander D, Reinert S and Hoffmann J: Prognostic value of c-Met expression in oral squamous cell carcinoma. Exp Ther Med 1: 69-72, 2010.

25. Hanna JA, Bordeaux J, Rimm DL and Agarwal S: The function, proteolytic processing and histopathology of Met in cancer. Adv Cancer Res 103: 1-23, 2009.

26. Kowalczuk O, Kozlowski M, Niklinska W, Kisluk J, Niklinska BJ and Niklinski J: Increased MET gene copy number but not mRNA level predicts postoperative recurrence in patients with non-small cell lung cancer. Transl Oncol 7: 605-612, 2014.

27. Singletary K and Ellington A: Genistein suppresses proliferation and MET oncogene expression and induces EGR-1 tumor suppressor expression in immortalized human breast epithelial cells. Anticancer Res 26: 1039-1048, 2006. 